\title{
Spoon versus knife
}

Cite as: CMAJ 2017 July 10;189:E915-6. doi: 10.1503/cmaj.161268

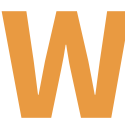

e were both shocked by the scratch marks on her back ... (Figure 1).

$10,11,12 \ldots$ the scratches on her back seemed to be intercostal and nearly anatomic. She was not moving her legs and she had urinary incontinence, and yet, she had been relying on having her back scratched with a spoon for the last three days. I was not aware of this form of traditional medicine, but my staff surgeon had seen it before. The treatment was not working. She would need immediate surgery.

T10, T11, T12 ... the dermal abrasions followed anatomic dermatomes. As a child, I had seen this form of treatment before. My grandmother (Ah-Pal) was quite skillful with her spoon. However, there is no arguing that cauda equina compression is a surgical emergency. Scratching the back with a spoon would not relieve the pressure from a massively herniated lumbar disc.

Gua Sha (dermal abrasion) employs a smooth object, usually a ceramic Chinese soup spoon, to scrape the lubricated skin along major meridians of the body until petechiae and ecchymosis show. ${ }^{1}$ These apparently nonpainful marks fade within a few days. It is thought that the scraping rebalances the meridians and releases the "bad wind" that is responsible for the ailment. These marks have sometimes been reported as signs of physical trauma, abuse or torture. ${ }^{2}$ As I looked at the patient lying prone for spinal surgery, it struck me as curious that an ancient method had left marks that so

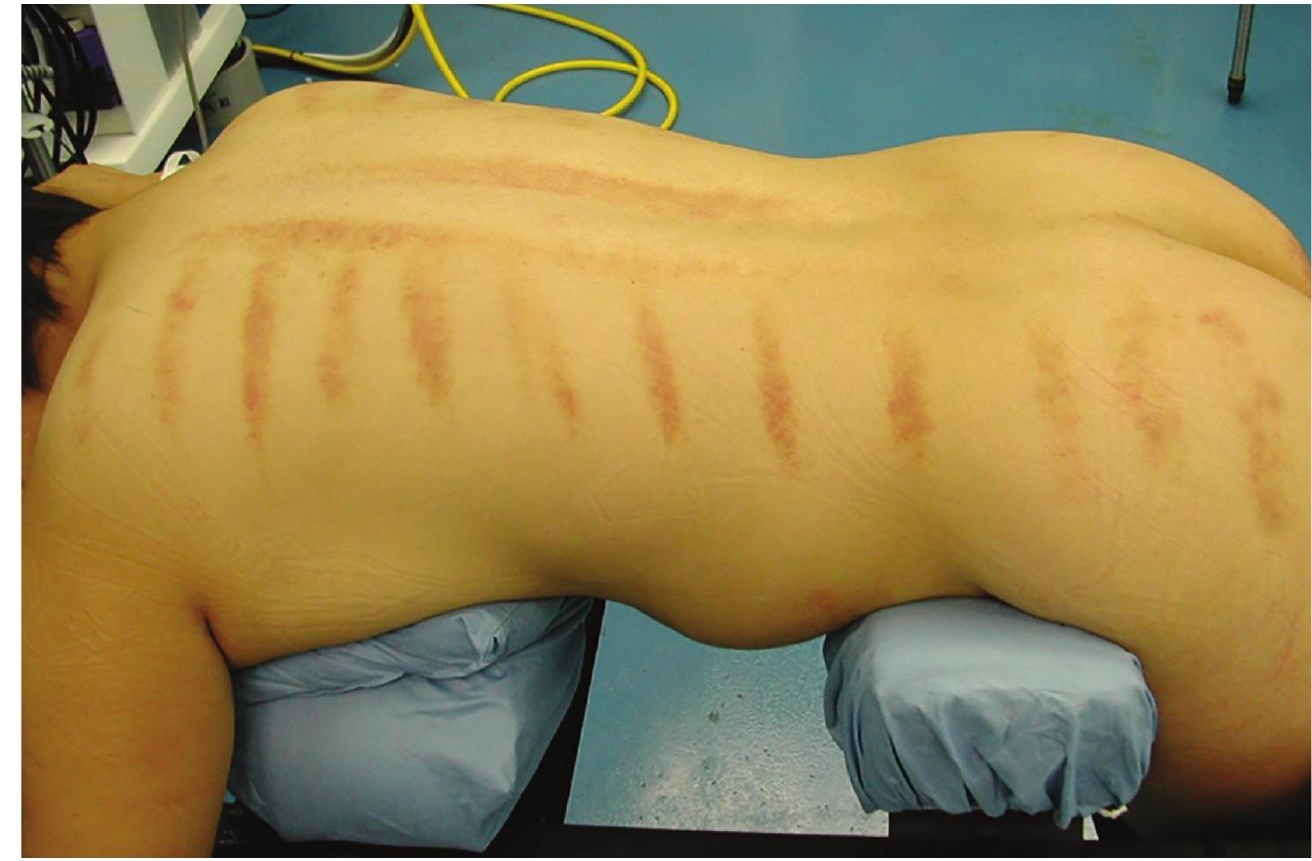

Figure 1: Gua Sha markings along the meridians of a woman's back.

clearly demarcated the dermatomes taught in modern Western medicine.

$0.5 \%$ twice daily, $0.5 \%$ three times daily, $1 \%$ three times daily ... the prescribed topical steroids and antihistamines were not working. My childhood eczema, which had been a nuisance when I was five, had become a notable inconvenience in my teenage years and now into my adulthood. These treatments seemed to work so well according to our medical school lectures last year.

Frustrated with the ineffectiveness of the prescribed medications, my parents brought me to see some other "medical consultants." I recall my parents brewing concoctions of dead bees mixed with fragrant herbs in a special clay pot and encouraging me to drink the black stew. Cleverly, they used Billy Bee Honey to sweeten the taste and turned the vile cocktail into another sugary drink of child- hood. When there were no enduring effects, my parents later consulted an acupuncturist to get rid of my eczema once and for all - or so they thought. There I was, lying in a dimly lit room for 20 minutes, hardly moving, with needles stuck all over my body. I still remember feeling each needle going in ... 28, 29, 30.

38, 39, 40 degrees Celsius ... my fever was not coming down. The pediatrician diagnosed the flu. But I was getting very sick. I also recall my parents bringing me to see more "medical consultants." They could make a diagnosis just by feeling my radial pulse, or looking at the colour of my sclera and my tongue. I must have been tachycardic but without jaundice or fetor hepaticus. I also recall my parents brewing concoctions in a clay pot and "strongly encouraging" me to drink the black stew. There was no honey in the stew, since bit- 
ter medicine always works better: the more bitter, the better!

It was typhoid fever. I was delirious and septic when my Ah-Pal insisted on bringing me to the hospital. My temperature finally came down ... 39, 38, 37.

Both of us were brought up in cultures that use traditional medicine, and yet we were taught Western medicine, its science and evidence. Seeing Gua Sha on our patient's body made us think about our own experience with traditional medicine. We are torn about its validity as we weigh the irresolute evidence against our parents' resolute determination that traditional medicine has passed the test of time. Our patient's Gua Sha did not work, but can we discount all traditional medicine? Are traditional medicine and Western medicine mutually exclusive? East versus West. Spoon versus knife.

Myself, I am at an impasse. The herbal medications and the acupuncture have had no lasting impact on my daily itchiness and dry skin. Nonetheless, whether it is a topical steroid or antihistamine, I have yet to find any luck with Western medicine, either.
On the other hand, I had better luck with Western medicine. Ah-Pal always credited Western medicine with saving my life. Yet, she remained an ardent believer in traditional medicine even in her final days.

Why do patients turn to traditional medicine, alternative medicine, or nonevidence-based treatments? Is it due to cultural practices or ancestral customs? For us, we had no choice: our parents have always been believers. Is it due to lack of faith in Western medicine? Do they feel that Western medicine has too many barriers to timely care? Is it out of desperation or hopelessness? And yet, it was out of desperation that our patient and my grandmother turned to Western medicine for critical care, and access to timely care did not seem to be an issue. On the other hand, hopelessness and desperation have driven many patients with incurable diseases to alternative medicine instead.

We do not have answers to many of these questions. We are not taught allopathic medicine, even in an age of cultural diversification and globalization. However, before Western medicine can decide to accept and incorporate - or denigrate and discourage - complementary medicine, research must be done to determine harm and benefit.

Our patient did well after surgery, with a complete recovery, although how she perceived her surgical care, we do not know. When she has another illness, will she have her body scraped with spoons again until it turns red? Has being put under the knife changed her beliefs about meridians? How much of her improvement will she attribute to my knife versus her spoon? 50\%, 75\%, 100\%?

\section{Han Yan BA}

Medical student

\section{Wai P. Ng MD}

Neurosurgeon

Western University, London, Ont.

\section{References}

1. Odhav A, Patel D, Stanford CW, et al. Report of a case of Gua Sha and an awareness of folk remedies. Int J Dermatol 2013;52:892-3.

2. Stewart GM, Rosenberg NM. Conditions mistaken for child abuse: Part II. Pediatr Emerg Care 1996; 12:217-21.

This article has been peer reviewed.

This is a true story. The authors have obtained patient consent. 